\title{
Sender-Based TCP Scheme for Improving Performance in Wireless Environment
}

\author{
Jahwan Koo, Sung-Gon Mun, and Hyunseung Choo* \\ School of Information and Communication Engineering, Sungkyunkwan University \\ Chunchun-dong 300, Jangan-gu, Suwon 440-746, South Korea \\ jhkoo@songgang.skku.ac.kr, \{msgon, choo\}@ece.skku.ac.kr
}

\begin{abstract}
Conventional TCP in wireless environment has a lack of ability to differentiate the packet losses caused by network congestion from those caused by wireless link error. When the packet losses are due to wireless link error, TCP performance degradation occurs because of its congestion control mechanism. This paper propose a sender-side TCP scheme for discriminating the cause of packet losses. Simulation results show that it outperforms existing TCP congestion mechanisms such as WestwoodNR, TCP New Jersey, and TCP Reno in terms of goodput performance in wireless links.
\end{abstract}

Keywords: TCP, congestion control, wireless environment.

\section{Introduction}

In wireless environment, conventional TCP is unable to differentiate the cause of packet losses, resulting in severe performance degradation [1. Many researchers attempt to improve TCP performance in wireless networks 2. Current research work has been classified into two directions: the end-to-end TCP modifications such as congestion control mechanism and link layer approaches that include intermediate router mechanisms. However, TCP modifications do not distinguish the cause of packet loss whether it is based on network congestion or wireless link error. Link layer approaches require more time and cost to be deployed in real wired and wireless networks. Therefore, discrimination of the cause of packet loss using the modified end-to-end TCP mechanism, without supporting the intermediate router mechanisms, is very important.

This paper propose WestwoodVT (WestwoodNR based on TCP Vegas buffer Thresholds), a sender-side TCP scheme, which discriminates the cause of packet loss and operates according to the cause when packet retransmission is required. It uses the flow control concept of TCP Vegas 3. so as to discriminate the cause. If the cause is due to network congestion, it retransmits packets using the available bandwidth estimator of WestwoodNR [4, which is an efficient TCP scheme in wired and wireless networks. Meanwhile, if the cause is due to wireless link error, it ignores the loss and responses as if the packet loss has never been occurred.

\footnotetext{
^ Corresponding author.
} 


\section{Discriminating the Cause of Packet Loss}

In this section, we present a novel scheme for improving TCP performance in wireless environment. WestwoodVT investigates the buffer state of network nodes between sender and receiver in order to discriminate the cause of packet loss. The discrimination inherits from the concept of TCP Vegas. WestwoodVT estimates the maximum Expected transmission rate and Actual transmission rate when received every ACK. The Expected transmission rate is given by where WindowSize is the size of the current congestion window, equal to the number of bytes in transit, BaseRTT is the minimum value of all measured RTT. In addition, Actual transmission rate is given where WindowSize is equal to WindowSize of Equation 1, RTT is the current calculated RTT.

$$
\text { Expected }=\frac{\text { WindowSize }}{\text { BaseRTT }}, \text { Actual }=\frac{\text { WindowSize }}{R T T}
$$

Additionally, WestwoodVT defines $\triangle$ is calculated by the difference of Actual from Expected transmission rate. The value of $\triangle$ presents the amount of current existing data in buffer of network nodes. Namely, $\triangle$ indicates the state of the current network. This is given by:

$$
\triangle=\left(\frac{\text { WindowSize }}{\text { BaseRTT }}-\frac{\text { WindowSize }}{R T T}\right) \times \text { BaseRTT }
$$

In addition, WestwoodVT defines the buffer thresholds, $\alpha$ and $\beta$. These values indicate to the lower and upper bound of network nodes's buffer. The discrimination of the cause of packet loss is operated in congestion avoidance (CA) phase. In CA phase, the sender estimates $\triangle$ using Eq. 1 , and 2 when it receives 3-DUPACK. WestwoodVT then compares $\triangle$ to the buffer thresholds $\alpha$ and $\beta$.

If $\triangle$ is smaller than the threshold $\alpha$, WestwoodVT assumes that the current buffer state of network nodes is loose, and decides that the packet loss is due to wireless link error. If $\triangle$ is greater than the threshold $\beta$, the current buffer state is tight. At this point, WestwoodVT assumes that the packet loss is caused by network congestion. If $\triangle$ is greater than $\alpha$ and smaller than $\beta$, WestwoodVT does not decide the cause of the packet loss. Due to the ambiguousness of the discriminating, WestwoodVT maintains the current state and then retransmits lost packets as the standard sending. Therefore, WestwoodVT postpones the decision of cause to the next it receives 3 -DUPACK for the more accurate discriminating.

\section{Performance Evaluation}

The simulation environments are depicted in Figure 1(a) and Figure 1(b), In Figure 1(a) a single TCP connection running a long-live FTP application delivers data from sender to receiver, with various wireless link errors: $0.1 \%, 1 \%, 2 \%$, $3 \%, 4 \%$, and $5 \%$, respectively. In Figure 1(a), all network nodes retain the queue size of 20 MSS. Furthermore, we construct a more complex topology, such as Figure 1(b) In this case, all node's queues are also set to 20 MSS. A single TCP 
connection running a long-live FTP application delivers data from the sender to the receiver with various wireless link errors: $0.1 \%, 1 \%, 2 \%, 3 \%, 4 \%$, and $5 \%$, respectively. At the same time, cross-traffic flows are generated: forward traffic flows from node1 to node 2 via router1 and base station, and backward traffic flows from node 3 to node 4 via base station and router1, respectively.

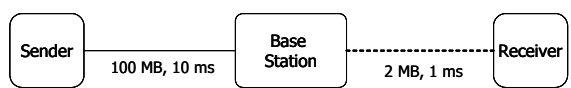

(a) Simulation topology

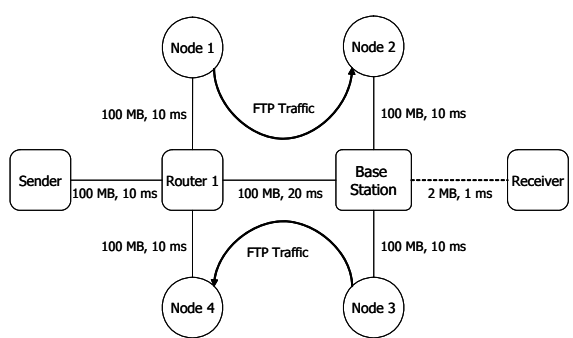

(b) Complex topology with background traffic

Fig. 1. Simulation topology

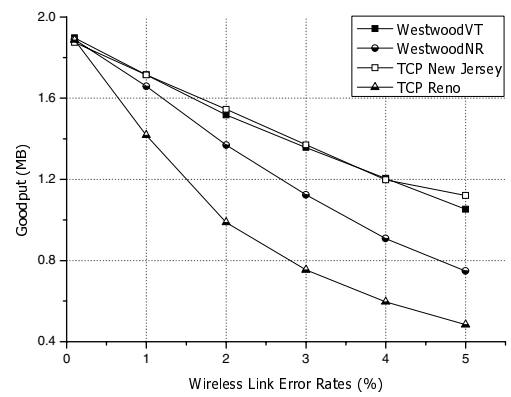

(a) Wireless link error rates

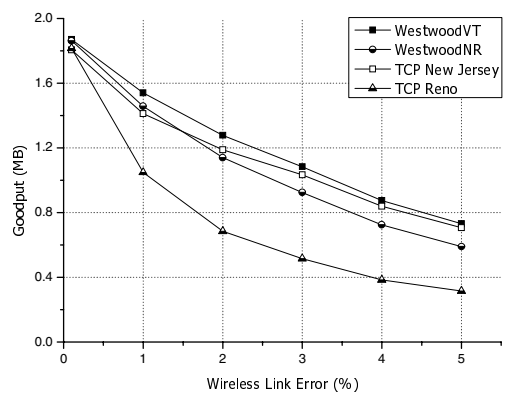

(b) Wireless link error rates with background traffic

Fig. 2. Goodput performance

We setup the lower bound of WestwoodVT, $\alpha$ to 14 . if $\triangle<14$, the cause is due to wireless link error. The upper bound, $\beta$ is fixed on 16, if $\triangle>16$, the cause is due to network congestion. Finally, we run the simulation for WestwoodVT, WestwoodNR, TCP New Jersey, and TCP Reno, respectively. Figure 2(a) demonstrates the result of simulation, an average of 10 times, over 200 seconds.

As the results of the first simulation, WestwoodVT is superior over WestwoodNR and TCP Reno in every wireless link error rates. With wireless link error rate of 1\%, WestwoodVT outperforms WestwoodNR by $3 \%$ and TCP Reno by $21 \%$. WestwoodVT outperforms WestwoodNR by $11 \%$ and TCP Reno by $54 \%$ 
with wireless link error rate of $2 \%$. Further, WestwoodVT is almost identical to TCP New Jersey, until the wireless link error rate reaches 4\%. WestwoodVT achieves a maximum of $41 \%$ and $118 \%$ improvements in goodput over WestwoodNR and TCP Reno, in various wireless link errors rates, respectively.

Figure 2(b) depicts results of the second simulation over 200 seconds, an average of 10 times. The buffer thresholds, $\alpha$ and $\beta$ of WestwoodVT are configured to 14 and 16, respectively. These values are based on the queue size of network nodes. The performance of TCP Reno is worst. WestwoodVT achieves $6 \% \sim$ $24 \%$ improvements in goodput over WestwoodNR, and it is overwhelming TCP New Jersey, with every wireless error rates. In this simulation, TCP New Jersey experiences the decreasing of throughput with background traffics. However, WestwoodVT keeps its performance constantly, regardless of wireless link error rates or background traffics.

\section{Conclusion}

In this paper, we have proposed WestwoodVT which utilizes the sender-based transmission window control mechanism for discriminating the cause of packet loss. It checks the buffer state of network nodes between sender and receiver using TCP Vegas operating mechanism and discriminates the cause of packet loss based on the buffer state. When the packet loss is due to network congestion, WestwoodVT uses the available bandwidth estimator of WestwoodNR for packet retransmissions. Otherwise, it retransmits packets without congestion control mechanism.

\section{Acknowledgements}

This research was supported by the MIC, Korea, under the ITRC support program supervised by the IITA, IITA-2006-(C1090-0603-0046).

\section{References}

1. Xylomenos, G., Polyzos, G. C.:TCP Performance Issues over Wireless Links. IEEE Communications Magazine, Vol. 39. (2001) 52-58

2. Tian, Y., Xu, K., Ansari, N.:TCP in Wireless Environments: Problems and Solutions. IEEE Radio Communications, Vol. 43. (2005) S27-S32

3. Brakmo, L. S., O’Malley, S. W., Peterson, L. L.:TCP Vegas: New Techniques for Congestion Detection and Avoidance. ACM/SIGCOMM Computer Communication Review, Vol. 24, (1994) 24-35

4. Casetti, C., Gerla, M., Mascolo, S., Sanadidi, M. Y., Wang, R.:TCP Westwood: Bandwidth Estimation for Enhanced Transport over Wireless Links. ACM/Mobicom, (2001) 287-297 